54. Seward, F., Burginyon, G. A., Grader, R. J., Hill, R. W., Palmieri, T. M. 1972, Ap. J., 178, 131.

55. Schwartz, D. A. $\quad 1970$, Ap. J., 162, 439.

56. Schwartz, D. A., Hudson, H. S., Peterson, L. E. $\quad$ 1970, Ap.J., 162, 431.

57. Peterson, L. E. 1972, IAU Symp. 55, 51.

58. Lewin, W. H. G., McClintock, J. E., Smith, W. B. $\quad$ 1970, Ap. J. (Letters), 159, L193.

59. Lewin, W. H. G., McClinton, J. E., Ryckman, S. G., Smith, W. B. 1971 , Ap. J. (Letters), 166, L69.

60. McClintock, J. E., Ricker, G. R., Lewin, W. H. G. 1971, Ap. J. (Letters), 166, L73.

\title{
2. GAMMA-RAY ASTRONOMY
}

\section{G. G. Fazio}

Over the years, gamma-ray astronomy has advanced rapidly, yet it has not enjoyed the spectacular surprises and successes of X-ray astronomy, particularly with respect to the intensity and number of sources detected. In the late 1950's the theoretical outlook for intense gamma-ray sources was promising, but as more sensitive detectors were developed, the early optimistic predictions vanished. Despite the difficulties associated with gamma-ray astronomy, considerable activity still exists and positive results have been produced.

The first evidence for the detection of a gamma-ray flux came from the results of the counter telescope on the OSO-3 satellite (Clark et al. (1)). Recently, a final summary of their results has been published (Kraushaar et al. (2)). The experiment detected gamma rays above $50 \mathrm{MeV}$ from the Galaxy, concentrated in a band of directions around the galactic equator with a broad maximum toward the galactic center. The equivalent line-source intensity at the center was $1.3 \times 10^{-4}$ photon $\mathrm{cm}^{-2} \mathrm{~s}^{-1} \mathrm{rad}^{-1}$. The gamma-ray intensity away from the galactic center is consistent with a production by $\pi^{0}$-meson decay, the $\pi^{0}$ mesons being produced by high-energy cosmic-ray interactions with the interstellar gas (Cavallo and Gould (3), Stecker $(4,5)$ ). The increase in intensity at the center cannot be explained by this mechanism, unless one assumes an increase in the primary cosmic-ray intensity. The fact that the gamma-ray distribution at the center is similar to the nonthermal radio emission suggests a mechanism involving cosmic-ray electrons, most probably Compton scattering. Electron scattering on the large submillimeter radiation (Shen (6), Cowsik and $\mathrm{Pal}(7)$ ) is sufficient, but the resulting X-ray intensity is too large. Balloon-flight experiments by Fichtel et al. (8), Bennet et al. (9), and Helmken (10) seem to have verified the results at the galactic center, and several other groups have evidence, of marginal significance, of gamma radiation from other regions of the galactic plane. However, a serious contradiction to these results has been the balloon-flight experiments of Frye $\mathrm{et} \mathrm{al}$. (11), who failed to detect any excess diffuse radiation above $50 \mathrm{MeV}$ from the galactic center and plane. The problem is complicated by the poor spatial resolution of the detectors, and by the fact that the flux value is obtained from the difference between two large numbers.

One of the most interesting developments over the past few years has been the possible detection of a number of discrete gamma-ray sources in the galactic plane, with some near the galactic center. In contrast to their negative results on a diffuse flux from the galactic center, Frye et al. (12) and Frye $(13,14)$ have reported evidence for several discrete sources in this region. In fact, two of the sources lie within the region of galactic-center emission reported by OSO-3. Their intensity $\left(\sim 10^{-5}\right.$ photon $\mathrm{cm}^{-2} \mathrm{~s}^{-1}$ ) is such that they could not have been detected by OSO-3; however, it should be noted that many more discrete sources would be needed to explain the measured OSO-3 flux. Browning et al. $(15,16)$ detected three sources in the galactic plane, near the center, and each was associated with an X-ray source. Three of the six most significant sources found by Frye's group may also be associated with X-ray sources. Dahlbacka et al. (17) also reported evidence for three discrete sources in this region, but only one was in the galactic plane.

The Cygnus region of the galactic plane is another region where discrete sources and enhanced emission have been reported (Browning et al. (15), Niel et al. (18)). Dean et al. (19) have also reported evidence for $\mathbf{0 \cdot 8 -}$ to $10-\mathrm{MeV}$ gamma rays from the region of Cygnus $\mathrm{X} 2$ and $\mathrm{X} 4$. At energies above 
$10^{12} \mathrm{eV}$, the Crimean Astrophysical Observatory (Vladimirsky et al. (20), Stepanyan et al. (21)) reported sources in Cygnus and Cassiopeia. The Cygnus source was not seen by the Smithsonian Astrophysical Observatory group at $10^{11} \mathrm{eV}$ (Weekes (22)).

In the anticenter region Volobuyev et al. (23), with a counter telescope on the satellites Cosmos 251 and 254, detected a large gamma-ray flux from the variable Seyfert galaxy 2C 120, during a period of maximum radio intensity. The Saclay-Milan-Palermo group (Forichon et al. (24)) has reported evidence for seven discrete sources in the vicinity of the galactic disk in the anticenter region.

There is no doubt that the galactic plane and the region near the galactic center are sources of gamma radiation above $10 \mathrm{MeV}$. What the source of this radiation is -i.e., whether it is diffuse and interstellar in origin or a group of discrete sources - is still uncertain. In regard to the multitude of discrete sources reported, no source has been seen of a high statistical significance by two different groups. Thus the evidence for any discrete source, other than the Crab Nebula, is not conclusive. Time variability of the source intensity should also be considered.

It is interesting to note that the lower energy gamma-ray spectrum of the galactic center has been measured up to energies of the order of $1 \mathrm{MeV}$ and evidence has been found for a spectral-line feature at $0.5 \mathrm{MeV}$ due to electron-positron annihilation (Johnson et al. (25)).

The most observed object in gamma-ray astronomy has been the Crab Nebula and its associated pulsar 0532. Over the past three years the spectrum of NP 0532 has been extended from the X-ray region to energies of $10^{12} \mathrm{eV}$. Hillier et al. (26) first reported evidence for pulsed emission in the 0.6- to 9-MeV region that was comparable to or exceeded in intensity the extrapolated total X-ray spectrum. Kurfess (27) published convincing evidence for pulsed emission up to energies of $1 \mathrm{MeV}$, and noted that the ratio of pulsed to total emission in the 100 - to $400-\mathrm{keV}$ region was $42 \pm 12 \%$; the ratio rises from a value of $2 \%$ at $1 \mathrm{KeV}$. The ratio of the secondary to primary peak was $2 \cdot 3 \pm 0 \cdot 2$, and the pulsed-emission spectrum was given by $10 \mathrm{E}^{-1.2} \mathrm{keV} \mathrm{cm}^{-2} \mathrm{~s}^{-1} \mathrm{keV}^{-1}$. Orwig et al. (28) simultaneously reported results consistent with those of Kurfess; both these result are an order of magnitude below those of Hillier.

In the region above $10 \mathrm{MeV}$, some confusion previously existed as to whether a pulsed emission from NP 0532 was detected. At the IAU Symposium No. 55 (Madrid) on X-Ray and Gamma-Ray Astronomy, several groups presented more convincing evidence for such a flux. The most significant result was that of Albats $e t$ al. (29). They reported that pulsed emission had been detected up to $100 \mathrm{MeV}$. The total pulsed intensity at $20 \mathrm{MeV}$ was $(9 \pm 4) \times 10^{-5} \mathrm{MeV} \mathrm{cm}^{-2} \mathrm{~s}^{-1} \mathrm{MeV}^{-1}$. Little emission was observed in the interpulse, in contradiction to the results at lower energies. The energy spectrum is of the form $\sim E^{-0.9}$, consistent with an extrapolation from X-ray energies. The pulsed energy flux in this region of $1.5 \mathrm{keV} \mathrm{cm}{ }^{-2} \mathrm{~s}^{-1}$ is comparable to the time-independent energy flux above $1 \mathrm{keV}$, indicating that all the energy emitted in the $10-$ to $100-\mathrm{MeV}$ region may be pulsed. Several other groups have also presented evidence for pulsed emission in this region (Kinzer et al. (30), Parlier et al. (31)) with overall agreement in intensities. Recently, the Cornell group (Greisen, 1972, private communication) has detected pulsed emission at 200 and $500 \mathrm{MeV}$, using a large-area gas-Cerenkov telescope. In the low-energy gamma-ray region, the continuous flux from the Crab Nebula - i.e., a nonpulsed flux -has been detected only up to energies of the order of $400 \mathrm{keV}$.

Ground-based instruments can be used to detect cosmic gamma rays above $10^{11} \mathrm{eV}$ by observing the atmospheric Cerenkov light bursts they produce. Using this technique, Grindlay (32) reported detection of pulsed gamma rays at $6 \times 10^{11} \mathrm{eV}$ from NP 0532 . The pulsed flux was $1.25 \times 10^{-11}$ photon $\mathrm{cm}^{-1}$. However, the ratio of the interpulse to primary pulse was $\sim 3 \cdot 5: 1$, which follows the trend observed at low gamma-ray energies, but is opposite to the effect observed in the 10 - to $100-\mathrm{MeV}$ region. The measured spectrum is consistent with an extrapolation from the $\mathrm{X}$-ray data. The University College, Dublin, and UKAERE, Harwell, group also found evidence for a pulsed flux at $2 \times 10^{12} \mathrm{eV}$, but the peak emission was not in phase with the optical signal.

Helmken (10), describing the results from the Smithsonian Astrophysical Observatory, found no significant pulsed emission at $1.5 \times 10^{11} \mathrm{eV}$. The upper limit to the pulsed flux was a factor of seven below an extrapolation from Grindlay's result, using an $E^{-1.5}$ spectrum. Unless the spectrum is 
very flat, there is a disagreement with Grindlay's results that needs to be resolved. If the SAO result is correct, the pulsed gamma-ray spectrum of NP 0532 has exhibited a break between $10^{9}$ and $10^{11} \mathrm{eV}$.

A continuous flux of gamma rays has been detected from the Crab Nebula at energies above $2.5 \times 10^{11} \mathrm{eV}$ (Weekes et al. (33), Fazio et al. (34)). Observations of the nebula over a 3-yr period indicate an average flux of $(4.4 \pm 1.4) \times 10^{-11}$ photon $\mathrm{cm}^{-1}$, corresponding to an emission of $6 \times 10^{33} \mathrm{ergs} \mathrm{s}^{-1}$. However, the flux appears to vary with time, with the most significant flux occurring 60 to 120 days after a major spin-up of the pulsar NP 0532. The increase was observed on three different occasions, and the total gamma-ray energy observed on each occasion was $\sim 10^{41}$ ergs, which is approximately equal to the energy in the pulsar spin-up.

In addition to gamma-ray emission from discrete sources, an isotropic diffuse component also exists, probably of extragalactic origin. Approximately three years ago, the spectrum above $20 \mathrm{keV}$ was characterized by a decreasing power-law spectrum with the following important features: a possible break in the spectrum at $40 \mathrm{keV}$, a possible flattening of the spectrum beyond $1 \mathrm{MeV}$, and the suggestion of a flux beyond $100 \mathrm{MeV}$. Recently, at the IAU Symposium No. 55, Pal (35) summarized the recent developments in the search for this diffuse component. The major result of recent developments in the search for this diffuse component. The major result of recent data is that one spectrum could be fitted from $20 \mathrm{keV}$ to $1 \mathrm{MeV}$, i.e. $25\left(E_{\mathrm{kev}}\right)^{-2.1} \mathrm{~cm}^{-2} \mathrm{~s}^{-1} \mathrm{sr}^{-1} \mathrm{keV}^{-1}$. It appears the 40-keV break resulted from an overestimated flux due to background corrections in the detectors (Manchanda et al. (36), Dyer and Morfill (37)). The flattening at $1 \mathrm{MeV}$ previously reported by the Ranger III and ERS-18 satellite experiments also appears to be due to instrumental background. Recent data on Apollo 15 and 16 flights by Arnold et al. (38), on the Cosmos satellites by Golenetski et al. (39), the balloon data of Daniel) et al. (40), and the theoretical calculations of Danjo (41) have shown that the flattening of the spectrum does not occur.

Above $5 \mathrm{MeV}$, the Apollo 15 and 16 experiments have extended the spectrum to $30 \mathrm{MeV}$, where the intensity is $5 \times 10^{-5}$ photon $\mathrm{cm}^{-2} \mathrm{~s}^{-1} \mathrm{sr}^{-1} \mathrm{MeV}^{-1}$. Balloon flights experiments by MayerHasselwander et al. (42) and Share et al. (43) gave result in essential agreement.

Above $50 \mathrm{MeV}$, the only data point is the integral flux value determined in the OSO-3 experiment (Kraushaar et al. (2)). If this value is correct, a steepening of the diffuse spectrum may occur at $50 \mathrm{MeV}$, although the data are not inconsistent with a power-law extrapolation from X-ray energies.

The theoretical work on the origin of this radiation favors a Compton-scattering mechanism between galactic-leakage electrons and the $2.7 \mathrm{~K}$ background radiation (Brecher and Morrison (44), Cowsik and Kobetich (45)). Stecker et al. (46) have proposed that the radiation between 1 and $50 \mathrm{MeV}$ may be due to cosmic ray collisions or matter-antimatter annihilation at large redshifts. A superposition of discrete extragalactic sources has not been ruled out as the origin of the diffuse radiation, but either a large cosmological factor to increase the number of sources or a large number of weak $\left(<10^{-7}\right.$ photon $\left.\mathrm{cm}^{-2} \mathrm{~s}^{-1}\right)$ sources are required. A review of the diffuse X-and gamma-ray flux has been given by Silk (47).

A definite omnidirectional flux of $0.51-\mathrm{MeV}$ gamma rays owing to the electron-positron annihilation line was also observed on the Apollo 15 and 16 flights. The intensity was $2 \times 10^{-2}$ photon $\mathrm{cm}^{-2} \mathrm{~s}^{-1}$.

Progress in gamma-ray astronomy advanced significantly in 1972 with the launching of two satellite experiments. In March 1972, the ESRO satellite contained an experiment called MIMOSA, which uses a vidicon spark chamber to search for gamma rays above $50 \mathrm{MeV}$. In November 1972, SAS-B a satellite devoted entirely to gamma-ray astronomy, was launched by NASA/Goddard Space Flight Center. The detector is a wire-grid spark chamber sentative to gamma rays above $25 \mathrm{MeV}$.

The gamma-ray spectrometer on the Apollo 15 and 16 flights has been used to map the sky in the $0 \cdot 1-$ to $0 \cdot 4-\mathrm{MeV}$ region by using the spacecraft as an occulting disk.

Future experiments include the ESRO COS-B satellite (1974) with a wire spark chamber and energy calorimeter. The threshold energy is $30 \mathrm{MeV}$.

The HEAO-B gamma-ray experiment, scheduled for 1976, is a joint undertaking of groups at NASA/GSFC, Stanford University, Grumman Aerospace Corporation, and the Max Planck Insti- 
tute in Munich. The instrument will consist of a large-area digitized spark chamber and a crystal absorption spectrometer.

It is interesting to note that present-day balloon-borne detectors have sensitivities of the order of $10^{-5}$ photon $\mathrm{cm}^{-2}$ for discrete sources, whereas future satellite experiments will increase the sensitivity to $10^{-6}$ to $10^{-7}$ photon $\mathrm{cm}^{-2} \mathrm{~s}^{-1}$. However, it is possible to increase the sensitivity of future balloon-borne detectors, i.e., a $1-\mathrm{m}^{2}$ spark chamber flown at a pressure of $0.5 \mathrm{mb}$ and with a cut-off rigidity of $12 \mathrm{GV}$ will have a sensitivity of $\sim 10^{-6}$ photon $\mathrm{cm}^{-2} \mathrm{~s}^{-1}$.

Additional details on current activity and techniques in gamma-ray astronomy are given in review articles by Fazio (48), the ESRO Gamma-Ray Astrophysics Colloquium (49), Frye (13), and the IAU Symposium No. 55 (Pal (34)), Fazio (50).

\section{REFERENCES}

1. Clark, G. W., Garmire, G. P., Kraushaar, W. L. $\quad$ 1968, Ap. J. Lett., 153, L203.

2. Kraushaar, W. L., Clark, G. W., Garmire, G. P., Borken, R., Higbie, P., Leong, C., Thorsos, T. 1972, Ap. J. 177, 341.

3. Cavallo, G., Gould, R. J. 1971, Nuovo Cimento 2, 77.

4. Stecker, F. W. $\quad$ 1970, Ap. Space Sci., 6, 377.

5. Stecker, F. W. 1971, 'Cosmic Gamma Rays', NASA SP-249, Washington, D.C.

6. Shen, C. 1969, Phys. Rev. Lett., 22, L568.

7. Cowsik, R., Pal, Y. 1969, Proc. 11th. Intl. Conf. Cosmic Rays, Budapest.

8. Fichtel, C. E., Hartman, R. C., Kniffen, D. A., Sommer, M. 1972, Ap. J., 71, 131.

9. Bennett, K., Penengo, P., Rochester, G. K., Sanderson, T. R., Sood, R. K. $\quad$ 1972, Nature, 238, 31.

10. Helmken, H. 1972, IAU Symp. 55, Madrid.

11. Frye, G. M., Staib, J., Zych, A., Hopper, V., Rawlinson, W., Thomas, J. $\quad$ 1969, Nature, 223, 1320.

12. Frye, G. M., Staib, J., Zych, A., Hopper, V., Rawlinson, W., Thomas, J. $\quad$ 1971, Nature, 231, 372.

13. Frye, G. M., Jr. $1971,12 t$ th Intl. Conf. Cosmic Rays, Hobart, Tasmania, August.

14. Frye, G. M., Jr. 1972, IAU Symp. 55.

15. Browning, R., Ramsden, D., Wright, P. J. 1972a, Nature, 235, 128.

16. Browning, R., Ramsden, D., Wright, P. J. $\quad 1972 b$, Nature, 238, 138.

17. Dahlbacka, G. H., Freier, P. S., Waddington, C. J. 1972, preprint.

18. Niel, M. et al. 1972, Ap. J., 171, 529.

19. Dean, A., Gerardi, G., De Martinis, C., Monastero, G. F., Russo, A., Scarsi, L. 1972, preprint.

20. Vladimirsky, B. M., Povlov, I. V., Stepanyan, A. A., Fomin, V.P. $\quad$ 1971, 12th Intl. Conf. Cosmic Rays, Hobart, Tasmania, August (paper G-25).

21. Stepanyan, A. A., Vladimirsky, B. M., Fomin, V. P. $\quad 1972$, preprint.

22. Weekes, T. C. 1972, IAU Symp. 55, Madrid.

23. Volobuyev, S. A., Galper, A. M. Iyudin, A. F., Kirillov-Ugryumov, V. G., Luchkov, B. I., Ozerov, Yu. V. 1971, (12th Intl. Conf. Cosmic Rays, Hobart, Tasmania, August (paper OG-19).

24. Forichon, M., Agrinier, B., Leray, J. P., Parlier, B., Boella, G., Maraschi, L., Buccheri, R., Robba, N. R., Scarsi, L. 1972, preprint.

25. Johnson, W. N., III, Harnden, F. R., Jr., Haymes, R. C. $\quad$ 1972, Ap. J. Lett., 172, L1.

26. Hillier, R. R., Jackson, W. R., Murray, A., Redfern, R. M., Sale, R. J. $\quad 1970$, Ap. J. Lett., 162, L177.

27. Kurfess, J. D. $\quad 1971$, Ap. J. Lett., 168, L39.

28. Orwig, L. E., Chupp, E. L., Forrest, D. J. 1971, Nature, 231, 171.

29. Albats, P., Frye, G. M., Jr., Zych, A. D., Mace, O. B., Hopper, V. D., Thomas, J. A. 1972 , preprint.

30. Kinzer, R. L., Share, G. H., Seeman, N. 1973, Ap. J., (in press).

31. Parlier, P., Vasseur, J., Leray, J. P., Forichon, M., Agrinier, B., Boella, G., Maraschi, L., Buccheri, R., Sacco, B., Scarsi, L. 1972 , preprint.

32. Grindlay, J. E. 1972, Ap. J. Lett., 174, L9.

33. Weekes, T. C., Fazio, G. G., Heimken, H. F., O'Mongain, E., Rieke, G. H. $\quad$ 1972, Ap. J., 174, 165.

34. Fazio, G. G., Helmken, H. F., O'Mongain, E., Weekes, T. C. 1972, Ap. J. Lett., 175, L117.

35. Pal, Y. 1972, IAU Symp. 55, Madrid.

36. Manchanda, R. K., Danjo, A., Sreekantan, B. V. 1972, preprint.

37. Dyer, C. S., Morfill, G. E. $\quad$ 1971, Ap. Space Sci., 14, 243. 
38. Arnold, J. R., Peterson, L. E., Metzger, A. E., Trombka, J. I. 1972, IAU Symp. 55, Madrid. 39. Golenetskii, S. V., Mazets, E. P., Ilinskii, V. N., Aptekar, R. L., Dredov, M. M., Guruyan, Yu. A., Panov, V. N. 1971, Ap. Lett., 9, 69.

40. Daniel, R. R., Joseph, G., Lavakare, P. J. 1972, preprint.

41. Danjo, A. 1972, preprint.

42. Mayer-Hasselwander, H. A., Pfeffermann, E., Pinkau, K., Rothermel, H., Sommer, M. 1972, Ap. J. Lett., 175, L23.

43. Share, G. H., Kinzer, R. L., Seeman, N. 1972, preprint.

44. Brecher, K., Morrison, P. 1969, Phys. Rev. Lett., 23, 802.

45. Cowsik, R., Kobetich, E. J. 1971, Proc. 12th Intl. Conf. Cosmic Rays, Hobart, Tasmania, August.

46. Stecker, F. W., Morgan, D. L., Jr., Bredekamp, J. $\quad$ 1971, Phys. Rev. Lett., 27, 1469.

47. Silk, J. 1970, Space Sci. Rev., 11, 671.

48. Fazio, G. G. 1970, Nature, 225, 905.

49. European Space Research Organization, 1970, Proc. ESRO Colloq. on Gamma-Ray Astrophysics, Noordwijk, The Netherlands.

50. Fazio, G. G. 1972, IAU Symp. 55, 303.

\section{COSMIC RAYS: PARTICLE ASTRONOMY}

\section{Maurice M. Shapiro, Rein Silberberg}

Cosmic-ray particles provide a probe for investigating the interstallar medium and high-energy processes in astrophysics. There have been noteworthy advances in cosmic-ray research during the last three years, subsequent to the publication of several comprehensive reviews, e.g., those of Hayakawa (1) and Meyer (2) giving overviews of the field, Daniel and Stephens (3) on cosmic-ray electrons, Parker (4) on the dynamics of the cosmic-ray gas in the galaxy, and Shapiro and Silberberg (5) on cosmic-ray nuclei. The present brief summary of recent highlights has perforce omitted many significant developments. Details of recent research appear in the 500 papers $(6,7)$ presented at the 12th International Conference on Cosmic Rays held in Hobart, Tasmania in August, 1971.

\section{Observations of cosmic-ray nuclei: elemental and isotopic composition}

Charge resolution and the statistics of events have improved so that abundances of the elements from hydrogen to iron are now fairly well known $(\mathbf{5 , 8 - 1 3})$, and the isotopic composition of hydrogen and helium has been measured (14). On the other hand, the study of isotopic abundances for elements heavier than helium has barely begun $(15,16)$. New measurements have markedly improved our knowledge of some abundances, e.g., those of nickel and chromium.

There is a striking difference between the composition of cosmic rays and the general abundances (17) of elements and isotopes: the cosmic rays are greatly enriched in the fragmentation products of heavier nuclei. Examples of such fragmentation products are ${ }^{2} \mathrm{H},{ }^{3} \mathrm{He}, \mathrm{Li}, \mathrm{Be}, \mathrm{B}$ and elements with atomic numbers 17-25. The abundances of these nuclei imply that cosmic rays have passed through 4 to $5 \mathrm{~g} \mathrm{~cm}^{-2}$ of material before reaching Earth $(13,18)$. Hence the cosmic rays seen to be confined for considerable times by galactic magnetic fields before leaking into intergalactic space.

Recent experiments $(\mathbf{1 0}, \mathbf{1 9})$, unlike some older ones, indicate that the fraction of fragmentation products is nearly independent of energy between $0 \cdot 1$ and $30 \mathrm{GeV} /$ a.m.u. (13). However, subsequent observations $(11,12)$ suggest that cosmic rays of higher energy $(30 \lesssim E \lesssim 100 \mathrm{GeV} /$ a.m.u. $)$ contain fewer fragmentation products: this higher energy component may have leaked out of the galaxy more readily. Further confirmation of these findings is essential; it would elucidate the nature of the hydromagnetic mechanisms that help to confine the galactic cosmic rays.

\section{Nuclear interactions with the interstellar gas}

As mentioned above, the cosmic rays we detect near Earth have traversed nearly $5 \mathrm{~g} \mathrm{~cm}^{-2}$ of material from the time of their initial production. As a result, about half of the nuclei between $\mathrm{C}$ and 